\title{
Norois
}

Environnement, aménagement, société

$250 \mid 2019$

Risque, littoral, paysage, port, géohistoire

\section{Les paysages creusois, entre nature réelle et nature rêvée}

A study of the emblematic landscapes of Creuse: real or dreamed nature?

\section{Raphaël Berthold}

\section{(2) OpenEdition}

1 Journals

\section{Édition électronique}

URL : https://journals.openedition.org/norois/7264

DOI : 10.4000 /norois. 7264

ISBN : 978-2-7535-7873-9

ISSN : $1760-8546$

\section{Éditeur}

Presses universitaires de Rennes

Édition imprimée

Date de publication : 19 juin 2019

Pagination : 7-26

ISBN : 978-2-7535-7871-5

ISSN : 0029-182X

\section{Référence électronique}

Raphaël Berthold, «Les paysages creusois, entre nature réelle et nature rêvée », Norois [En ligne], 250 |

2019, mis en ligne le 01 janvier 2022, consulté le 14 janvier 2022. URL : http://

journals.openedition.org/norois/7264 ; DOI : https://doi.org/10.4000/norois.7264

\section{(c) Tous droits réservés}




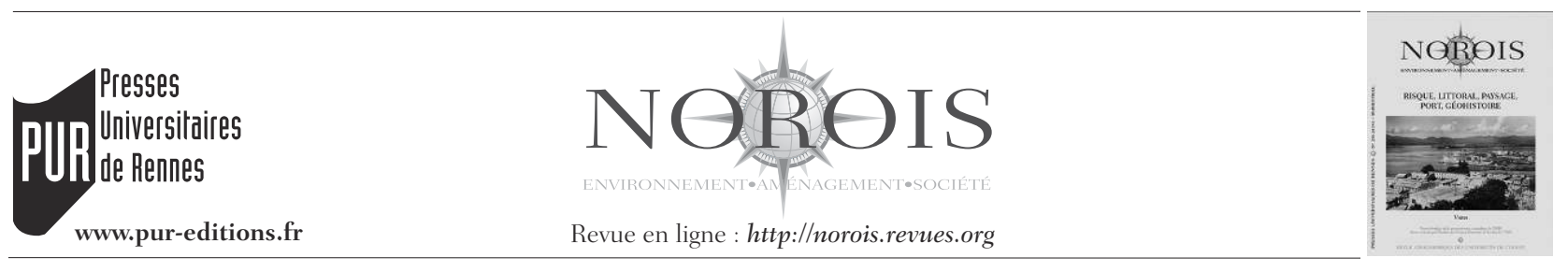

\title{
Les paysages creusois, entre nature réelle et nature rêvée
}

\author{
A Study of the Emblematic Landscapes of Creuse: \\ Real or Dreamed Nature?
}

\section{Raphaël BerTHOLD}

\author{
Université Clermont-Auvergne, UMR Territoires - 49, Boulevard François-Mitterrand, CS 60032, 63001 CLERMONT- \\ Ferrand Cedex 1, France. (raphael.berthold@uca.fr)
}

\begin{abstract}
Résumé : Cet article interroge la question des valeurs emblématiques et symboliques des paysages. Dans quelle mesure pouvonsnous identifier des paysages représentatifs d'un ensemble plus vaste? Comment pouvons-nous agréger à quelques paysages des valeurs communes au point de rendre acceptable leur aspect réducteur par rapport à l'ensemble des possibles? Pour conduire cette réflexion, nous proposons de nous appuyer sur les résultats d'une étude menée pour le Conseil Départemental de la Creuse à des fins de développement touristique. Notre cheminement méthodologique abordera successivement les enjeux inhérents à l'objectivité et à la subjectivité paysagère. La question de l'objectivité sera traitée à partir des résultats d'une monographie dont la finalité était d'identifier les éléments identitaires de la Creuse. La subjectivité, quant à elle, sera étudiée à partir des résultats de deux enquêtes concernant la perception de la Creuse et de ses paysages, réalisées auprès des habitants creusois et non creusois. Cette dernière approche nous conduira à aborder la dimension multi-sensorielle et sensible du paysage.
\end{abstract}

Abstract: This article addresses the question of the emblematic and symbolic nature of landscapes. To what extent is it possible to identify certain specific landscapes as being representative of a greater whole? How can a set of common traits be assigned to a few individual landscapes in such a way as to overcome the reductive aspect of this process in relation to the whole? To try to answer these questions, we propose to base our reflection on the conclusions of a study carried out on behalf of the Conseil Départemental de la Creuse (the local authority for the Creuse département, in central France) for purposes of touristic development. Our approach will involve considering the ins and outs of both objective and subjective perceptions of landscapes. The question of objectivity will be considered using the results of a case study carried out to pinpoint the different characteristics that make up the identity of the Creuse département. And subjectivity will be studied using the results of two surveys, conducted among locals and non-locals, about their personal perceptions of Creuse and its landscapes. This second approach will require us to examine the multisensorial and sense-related aspects of landscapes.

Mots clés : paysages - enquête - expérience géographique - paysages multisensoriels - Creuse

Keywords: landscapes - surveys - geographical experience - multi-sensory landscape - Creuse

«Nous semblions aller du savoir au sensible pour l'enrichir : en fait un mouvement d'une plus grande nécessité nous entraîne du sensible au savoir et à l'imaginaire » (Sansot, 1983).

\section{INTRODUCTION}

La loi française du 8 janvier 1993 sur la protection et la mise en valeur des paysages annonce à l'échelle nationale les enjeux de la Convention européenne 
du paysage de Florence de 2001 qui entre en vigueur en France le $1^{\text {er }}$ juillet 2006. Parmi ses principaux objectifs figure la nécessaire prise en considération des paysages ordinaires dans les politiques d'aménagement. En effet, si la question du paysage a longtemps été réservée à la gestion et à la valorisation des sites « exceptionnels », la Convention insiste sur la manière dont notre environnement paysager influe sur la qualité de notre cadre de vie. La question du paysage, de part ses enjeux sociétaux, s'invite donc de plus en plus régulièrement chez nos élus qui cherchent à obtenir par la concertation avec les habitants, l'approbation de leur politique en matière d'aménagement et de valorisation paysagers.

Une recherche de consensus délicat à obtenir sur un sujet qui passionne et qui divise beaucoup à propos de la manière dont se construisent les perceptions et les valeurs paysagères.

Ces constructions ont été abondamment traitées durant ces quarante dernières années et, sans tendre à l'exhaustivité, plusieurs approches animent encore le débat aujourd'hui.

Parmi les théories référentes - tout en considérant que la question est abondamment traitée par d'autres disciplines telles que la géographie, la sociologie, l'anthropologie, la philosophie - figure la thèse de Roger dite de « l'artialisation » du paysage formulée en 1997. Sa démonstration établit clairement l'influence des arts dans la construction du concept de paysage et de ses représentations : l'artiste, par le pinceau et la plume, transforme le « pays » en «paysage ».

Cette démonstration servira de fondement à de nombreux travaux postérieurs présentant une filiation plus ou moins forte avec celle-ci. Pour certains auteurs, "le concept de paysage trouve sa généalogie dans l'art », la " création artistique » est au fondement de «la conscience paysagère " (Veyret, Lemaître, 1996) qui conduit au début du XxI ${ }^{\mathrm{e}}$ siècle à l'acception du terme de paysage tel que nous le connaissons. Ainsi, si le paysage agit sur notre sensibilité, nous ne devons pas oublier que la perception humaine est déjà construite, structurée, organisée et articulée (Jakob, 2008). D'autres auteurs, tout en reconnaissant les intérêts de l'approche historicoculturelle, proposent une approche qui place la société - et pas seulement l'artiste - au centre de la construction paysagère (Luginbühl, 2012).
Si ces deux visions continuent globalement à structurer le débat avec des auteurs qui privilégient plutôt l'une à l'autre, selon leurs entrées conceptuelles et disciplinaires, il n'en demeure pas moins qu'ils sont très nombreux à reconnaître les intérêts d'une approche transversale. Lorsque Roger nous dit que le paysage ne peut se réduire à un "géo-système » ou à un "écosystème », il semble effectivement nécessaire de dépasser cette approche réductrice et de considérer qu'il constitue " un ensemble de systèmes matériels biologiques, sociaux, économiques et politiques dont la complexité est accrue par la dimension esthétique, symbolique » (Luginbühl, 2012). Toutefois, ces valeurs, qu'elles soient d'origine artistique, esthétique ou encore socio-économique (aménagement, cadre de vie), se trouvent à chaque fois associées à un contexte socio-culturel et à son caractère évolutif. Ainsi, l'une des grandes difficultés à traiter des représentations du paysage semble relever de cette difficile assimilation/restitution de leurs valeurs passées, présentes, et à venir.

Dans ce contexte, il semble que les nouveaux enjeux liés à la crise environnementale et touchant à la dégradation de notre cadre de vie se répercutent fortement sur nos manières de prendre en compte le paysage. En effet, ces enjeux ne sont-ils pas plus « appréciés » aujourd'hui pour leurs attributs environnementaux et sensoriels (notamment les ambiances sonores fortement associées à la question du cadre de vie) que pour leurs références littéraires et picturales? Les espaces identifiés comme préservés ne deviennent-ils pas aujourd'hui et plus que jamais, par l'intermédiaire de leurs paysages, des lieux « emblématiques » venant nourrir un idéal de nature parfois éloigné de la réalité paysagère?

Nous proposons d'apporter quelques éléments de réponses sur ce sujet à partir des résultats d'une étude menée pour le Conseil Départemental de la Creuse qui souhaitait, dans le cadre de son Schéma Départemental de Développement Touristique, identifier les paysages emblématiques de son territoire en vue de sa valorisation.

Après quelques explications d'ordre méthodologique qui semblent nécessaires - afin que le lecteur puisse bien cerner les enjeux et les limites de notre démonstration - nous tenterons de mettre en évidence les divergences et les convergences entre les éléments constitutifs d'une réalité paysagère et ses perceptions. La dernière partie sera consacrée 
à une réflexion sur les paysages sensoriels avec les avantages et les limites inhérents à sa subjectivité.

\section{Approche méthodologique}

Le cahier des charges de la commande "définir cent points de vue pour la Creuse » a orienté notre définition du mot paysage à sa plus simple définition - c'est-à-dire une "étendue de terre qui s'offre à notre regard ( (Dictionnaire Larousse) - dans la mesure où les paysages sélectionnés devaient être présentés dans un ouvrage destiné à un large public. Le terme «emblématique » quant à lui relève d'un « objet concret destiné à symboliser une notion abstraite ou représenter une collectivité, un métier, une personne... » (Dictionnaire Larousse). Cette sélection de paysages et des lieux auxquels ils sont associés nécessite de préciser au préalable ce que nous entendons par lieux emblématiques et symboliques. Selon Debarbieux (1995) ces lieux qui font le territoire relèvent simultanément de l'ordre des matérialités, de l'ordre des significations et de l'ordre des symboles. La matérialité exprime les éléments physiques de l'espace géographique qui peuvent être décrits et inventoriés (biotique et abiotique), les significations donnent une valeur, une fonction aux lieux qui sont reconnus et identifiés socialement, les symboles quant à eux apportent une autre valeur, reconnue collectivement (Monnet, 2000) pouvant être identitaire (Di Méo, 2002) et qui dépasse le cadre du territoire auxquels ils sont associés. Notre démarche souhaitant être la plus large possible, nous avons pris en compte les différents niveaux d'interprétation cités précédemment. Notre première étape, basée sur un travail d'interprétation de la matérialité (monographie) et de la perception des paysages (enquêtes), a permis d'identifier une série d'éléments identitaires (sous forme de mots clefs) afin de guider nos choix paysagers. La seconde étape a été consacrée à la sélection des paysages en prenant en considération la question du sensible. Cette dernière question étant fortement abordée lors des étapes précédentes et par le commanditaire, une dernière partie interrogera l'existence de valeurs sensorielles communes.

\section{Des éléments identitaires pour guider nos prises de vue}

La sélection des mots clefs s'est faite à partir de deux études. Une première étude exhaustive du territoire a donné lieu à la rédaction d'une monographie présentant les principales caractéristiques du territoire conduisant à une lecture plutôt « objective » et géographique des paysages. Une seconde étude a été réalisée sur les représentations des paysages à partir de deux enquêtes, l'une consacrée aux habitants de la Creuse, l'autre consacrée aux personnes non creusoises, amenant cette fois-ci une lecture plus subjective des paysages.

La combinaison de ces deux approches présentait l'avantage de construire une sélection prenant en compte ces deux réalités, celle-ci devant être à la fois le reflet d'une histoire réelle avec ses signes et empreintes, et le reflet de paysages vécus par les habitants creusois mais aussi perçus par des personnes habitant à l'extérieur du département.

\section{La rédaction de la monographie}

Ce premier protocole devait aboutir à la rédaction d'une synthèse mettant en évidence les principales caractéristiques du département. Pour ce faire, notre démarche a pris en compte deux grands domaines ayant pu laisser des traces ou des signes visibles.

- Le premier concerne les éléments physiques et sociaux abiotiques. Les éléments géophysiques sont les témoins d'une longue évolution des temps géologiques et de leurs actions sur le modelé topographique. Leur analyse s'est portée tout particulièrement sur l'étude de la géographie physique : géologie, reliefs, hydrographie, climat. Les éléments sociaux révèlent l'histoire des hommes qui a également laissé des traces durables faisant du paysage un objet qui exprime une civilisation et qui peut ainsi être décrit et inventorié (Berque, 1984). Dans ce cas, ce sont des ressources traitant du passé des hommes et de leurs sociétés qui ont été étudiées (histoire, économie).

- L'autre domaine concerne les éléments biotiques qui recouvrent la surface terrestre, c'est-àdire la faune et la flore qui composent la biodiversité et donc la variabilité des organismes vivants en fonction des biotopes (Levèque, Mounolou, 2001). 
Les informations résultent de documents traitant de l'environnement et de ses écosystèmes.

Pour les deux domaines, de nombreuses informations ont été tirées de l'ouvrage "Creuse encyclopédie »(Editions Bonneton), mais aussi de l'étude «Paysages et architectures en Pays Ouest Creuse : lire, comprendre et interpréter », ainsi que de cartes issues de l'Atlas paysager de la région (Carte des paysages creusois et de l'occupation des sols) en vue de sélectionner nos mots clefs. Ces derniers ont été choisis par les étudiants et les enseignants et ont été ensuite validés par les représentants du territoire : élus, professionnels du CAUE, professionnels du tourisme (annexe 2), lors d'ateliers. Cette démarche participative regroupait environ 25 personnes issues du monde professionnel et politique.

Le second protocole, dédié à l'étude des perceptions paysagères, reposait sur la mise en œuvre de deux enquêtes destinées cette fois à l'ensemble de la population.

\section{Des enquêtes pour l'étude des perceptions paysagères}

La prise en compte de la perception a été réalisée à partir de deux enquêtes qui se sont déroulées au cours de la période 2012-2014. L'une à destination des habitants de la Creuse afin de qualifier le paysage vu de l'intérieur du département (346 personnes), et la seconde à destination des personnes extérieures pour identifier cette fois les regards externes (551 personnes). Les enquêtes ont été diffusées durant 8 mois sur internet sous forme de «Google questionnaire » et d'autres ont été réalisées sur le terrain afin d'affiner notre échantillon. La population creusoise a été informée de l'étude et du questionnaire par la presse (La Montagne), la radio locale (Radio Limousin) et la distribution de flyers. Les questionnaires étaient accessibles via un lien internet et par un QR code. Le questionnaire dédié aux habitants hors Creuse a été diffusé via internet par les services du département et les étudiants. Toutes les enquêtes ont fait l'objet d'un traitement identique qui a été supervisé par l'équipe enseignante et un ingénieur d'étude.

\section{Profil des enquêtés creusois}

Cette enquête a touché davantage les femmes $(59,38 \%)$ que les hommes $(40,62 \%)$ alors qu'elles représentent $51,3 \%$ de la population creusoise et les hommes 48,7\%. Les classes d'âge des individus les plus nombreux à avoir répondu sont les 45-59 ans (33,8 \% contre $22,3 \%$ pour la population départementale), suivis des $60-74$ ans $(27,5 \%$ contre $18,4 \%)$ et des 30-44 ans (26,3\% contre $17,1 \%)$. Concernant la répartition par catégories socioprofessionnelles (CSP), la catégorie « cadres, professions intellectuelles supérieures » est celle qui a le plus participé et représente $25,1 \%$ de l'échantillon alors qu'elle n'en représente que 3,7 \% dans le département (tableau 1). Cette catégorie culturellement plus connectée et peut-être concernée par ce sujet est donc très largement surreprésentée. Suivent les retraités avec $22,5 \%$ et les employés avec 19,9\%. Les ouvriers et les agriculteurs sont les CSP ayant le moins répondu au questionnaire avec $1,7 \%$ et 3,5\% de l'échantillon. Cependant, les agriculteurs sont relativement bien représentés comparativement à leur poids dans la population creusoise (5\%). En revanche, pour les artisans, commerçants et chefs d'entreprise, bien qu'il s'agisse de la CSP la moins fréquente sur le plan départemental $(3,5 \%)$, elle est surreprésentée dans le cas de l'enquête avec 8,7\% de l'échantillon, ce qui pourrait s'expliquer par la distribution de flyers dans de très nombreux commerces.

\section{Profils des enquêtés non creusois}

Comme pour la première enquête " La Creuse vue de l'intérieur », la majorité des répondants est de sexe féminin. Si les chiffres de l'enquête sont mis en parallèle avec ceux de la France métropolitaine, les hommes sont sous-représentés (40,1\% contre $48,4 \%$ dans la population française) et les femmes surreprésentées $(59,9 \%$ contre 51,6\%).

De même pour les âges, les 15-29 ans sont les plus nombreux à avoir répondu et représentent 45,7\% des enquêtés, contrairement aux personnes âgées de 75-89 ans qui ne représentent que 0,7\% des répondants. Ces deux classes d'âge sont respectivement surreprésentées et sous-représentées. En France, les 15-29 ans représentent 18,6\% de la population totale et les 75-89 ans ont une part égale à 8,1\%. 


\begin{tabular}{|l|c|c|c|c|}
\hline CSP & $\begin{array}{c}\text { Enquêtés } \\
\text { creusois }\end{array}$ & $\begin{array}{c}\text { Population creusoise } \\
\text { (Chiffre INSEE } \\
\text { 2009) }\end{array}$ & $\begin{array}{c}\text { Enquêtés } \\
\text { non creusois }\end{array}$ & $\begin{array}{c}\text { Population française } \\
\text { (Chiffre INSEE } \\
\text { 2009) }\end{array}$ \\
\hline Agriculteurs et exploitants & 3,5 & 5 & 1,5 & 0,9 \\
\hline $\begin{array}{l}\text { Artisans, commerçants, } \\
\text { chefs d'entreprise }\end{array}$ & 8,7 & 3,5 & 2,7 & 3,3 \\
\hline $\begin{array}{l}\text { Cadres et professions intellectuelles } \\
\text { supérieures }\end{array}$ & 25,1 & 3,7 & 26,3 & 8,7 \\
\hline Professions intermédiaires & 10,7 & 9 & 10 & 13,9 \\
\hline Employés & 19,9 & 15,2 & 18,9 & 16,6 \\
\hline Ouvriers & 1,7 & 12 & 0,9 & 13,5 \\
\hline Retraités & 22,5 & 39,3 & 10,5 & 16,2 \\
\hline $\begin{array}{l}\text { Autres personnes sans activité } \\
\text { professionnelle }\end{array}$ & 7,8 & 12,3 & 29 & $100 \%$ \\
\hline Total en \% & $100 \%$ & $100 \%$ & $100 \%$ & 16,9 \\
\hline
\end{tabular}

Tableau 1 : Répartition des enquêtés selon la CSP comparée à la population creusoise et nationale Socio-professional breakdown of the survey group, compared with the population of Creuse and the French population as a whole

Au sujet des catégories socio-professionnelles, les «cadres et professions intellectuelles supérieures » (tableau 1) sont les plus représentés $(29 \%$ contre $8,7 \%$ pour la France) suivis de près par les « autres personnes sans activité professionnelle » $(26 \%$ contre 16,9\%). Comme dans la première enquête, les ouvriers $(0,9 \%$ pour $13,5 \%)$ et les agriculteurs/ exploitants $(1,6 \%)$ sont les moins représentés. Hormis les catégories "employés » et « artisans, commerçants », l'enquête n'est pas représentative de la population nationale hors Creuse.

L'origine géographique des répondants touche toute la France (20 régions) et particulièrement les régions voisines : Auvergne (20,5\%), RhôneAlpes (16,7 \%), Île de France (9,4\%), Pays de Loire $(7,6 \%)$, Limousin $(6,5 \%)$ Aquitaine $(5,1 \%)$.

Bien que ces échantillons ne soient pas totalement représentatifs de la population du département et de la France, il s'est avéré que le nombre des questionnaires exploitables était suffisant afin d'apporter des éléments de réponse à notre sujet.

Pour conclure sur cette première phase méthodologique, nous pouvons dire que ce long travail de recherches documentaires et d'enquêtes a permis de définir une centaine de mots clefs. La décision a alors été de les regrouper en quelques catégories (annexe 2) afin de faciliter leur classement et d'assurer une représentation proportionnée de leurs sujets. La dimension sensible, très présente dans notre monographie et souhaitée par nos commanditaires, fera alors l'objet d'une attention particulière (annexe 1) et justifiait d'un parti pris en lien avec la nouvelle stratégie touristique du département (la Creuse espace de création, de contemplation et d'évasion).

À ce stade, nous devions également penser à une méthode permettant de sélectionner nos prises de vue définitives car le champ des possibles semblait alors illimité. Un autre système de sélection devait être mis en place.

\section{La sélection des paysages emblématiques}

La sélection des paysages s'est faite à partir de deux protocoles devant répondre à deux objectifs.

- Le premier protocole visait à organiser une prospection «scientifique » du territoire en divisant ce dernier en 14 dalles de 300 à 600 km. Il était illusoire d'envisager une prospection totale des lieux mais au moins de tenter de le faire de manière organisée tout en couvrant le plus d'espace possible et de veiller à une certaine équirépartition géographique des sites choisis, le projet étant de valoriser les paysages de tout le département. Si des prises de vue ont été présélectionnées en laboratoire par les étudiants et les enseignants (lieux spécifiques ou sujets) afin de guider nos pérégrinations, nous attendions aussi beaucoup des hasards des rencontres, des paysages eux-mêmes, mais aussi des habitants 
pour leurs conseils dans la localisation des lieux ou en partageant des sites privilégiés à leurs yeux. Lors de chaque prise de vue, une grille paysagère (annexe 1) était remplie afin de mémoriser le contexte de la prise de vue en insistant notamment sur son caractère polysensoriel : ressenti personnel, action des météores, autres sens mobilisés comme l'ouïe ou l'odorat. Plusieurs milliers de clichés étant réalisés, ces grilles seront précieuses afin de faciliter nos choix définitifs. Les photographies ont toutes été réalisées par les étudiants et avec l'avis de l'équipe pédagogique.

- Le second protocole devait s'assurer de la bonne représentativité des paysages du département (emblématiques) en les soumettant à une série de 4 tests (tableau 2).

Le premier test vérifiait à un premier niveau les correspondances avec les mots clefs issus de la première étape. Le second test, plus discriminant, devait révéler d'un lien fort avec plusieurs motsclefs (d'une ou plusieurs catégories). Le troisième test devait révéler une dimension sensible qui, au fil de l'étude, est devenue déterminante. Enfin, le dernier test était l'acceptation par le commanditaire des paysages proposés; aucune prise de vue n'ayant été refusée par celui-ci.

Nos démarches nous ont permis de sélectionner 85 sites/paysages pouvant être considérés comme révélateurs - selon notre protocole - des paysages creusois.
Malgré une démarche qui tentait de concilier les approches objectives et subjectives, il n'en demeure pas moins qu'elle reste assez peu représentative de la "réalité paysagère ». Une étude "scientifique » du paysage aurait nécessité une approche mesurée et quantifiée de l'occupation des sols, des typologies de paysages, mais aussi de la mise en place de protocoles rigoureux nécessaires à l'analyse des compositions paysagères; structures, symboles, motifs, angles de vue (Joliet et al., 2004), ce qui n'est pas le cas ici. L'essentiel de notre cheminement relève d'une approche sélective (mots clefs) et sensible reposant sur des valeurs très subjectives. Les résultats de notre sélection en sont l'illustration (annexe 2).

\section{LES PAYSAGES CREUSOIS : ENTRE RÉALITES ET PERCEPTIONS}

Le premier niveau d'analyse de la monographie conforte le caractère particulièrement structurant du relief dans l'organisation générale des paysages. Ses formes élémentaires déterminent à la fois la profondeur du champ visuel, ses lignes de fuite mais aussi de nombreuses autres composantes du paysage tel que le réseau hydrographique, la végétation, l'agriculture. Les enquêtes révèleront une représentation beaucoup plus précise et subjective des paysages permettant de dissocier l'image d'un cadre de vie privilégié - comme fondement d'une

\begin{tabular}{|c|c|c|c|c|}
\hline \multirow{2}{*}{$\begin{array}{l}\text { Phases } \\
\text { de la sélection }\end{array}$} & Test 1 & Test 2 & Test 3 & Test 4 \\
\hline & Paysage représentatif & $\begin{array}{c}\text { Paysage considéré comme } \\
\text { «emblématique » }\end{array}$ & $\begin{array}{c}\text { Sensibilité particulière sur } \\
\text { le terrain }\end{array}$ & $\begin{array}{l}\text { Validation par le comman- } \\
\text { ditaire et les participants }\end{array}$ \\
\hline $\begin{array}{l}\text { Critères } \\
\text { de la sélection }\end{array}$ & $\begin{array}{c}\text { Mise en relation avec } \\
\text { plusieurs mots clefs issus } \\
\text { de la monographie et des } \\
\text { enquêtes }\end{array}$ & $\begin{array}{c}\text { Forte relation avec } \\
\text { plusieurs mots-clefs } \\
\text { d'une même ou plusieurs } \\
\text { catégories }\end{array}$ & $\begin{array}{c}\text { Approche sensible rensei- } \\
\text { gnée à partir de la grille de } \\
\text { lecture } \\
\text { (annexe } 2)\end{array}$ & $\begin{array}{c}\text { Présentation et valida- } \\
\text { tion devant le comité de } \\
\text { pilotage }\end{array}$ \\
\hline $\begin{array}{l}\text { Exemple } \\
\text { pour la caté- } \\
\text { gorie habitat/ } \\
\text { population }\end{array}$ & $\begin{array}{c}\text { Village, faible densité, } \\
\text { rural, } \\
\text { néo-ruraux, } \\
\text { anglais, } \\
\text { exode, vacuité, } \\
\text { terre d'accueil, } \\
\text { accessible (financier), } \\
\text { vieillissement, désert, } \\
\text { refuge, vide, cocon, îlot }\end{array}$ & $\begin{array}{l}\text { Village, } \\
\text { rural, } \\
\text { exode, } \\
\text { terre d'accueil }\end{array}$ & $\begin{array}{l}\text { Analyse et prise de vue } \\
\text { sensible } \\
\text { Ecriture sensible } \\
\text { Ambiances sonores }\end{array}$ & $\begin{array}{c}\text { - Village de Mazeirat } \\
\text { inhabité (exode) } \\
\text { - Square des Justes (terre } \\
\text { d'accueil) } \\
\text { - Ligne ferroviaire aban- } \\
\text { donnée Guéret/ Châtre } \\
\text { (exode) } \\
\text { - Pompes à essence soli- } \\
\text { taires (exode) }\end{array}$ \\
\hline
\end{tabular}

Tableau 2 : Sélection des paysages emblématiques A selection of emblematic landscapes 
forme d'archétype paysager pour les locaux - d'une image plus duale pour les non-locaux où l'omniprésence des ressources naturelles se trouve être aussi l'expression d'une fragilité économique.

\section{Les éléments identitaires issus de la monographie}

Les mots clefs issus de la monographie ont mis en évidence une double organisation des composantes paysagères.

- Une organisation physique qui découpe le territoire en trois unités de reliefs. Une partie peu élevée qui s'étend sur l'axe « La Souterraine-Guéret-Evauxles-Bains » (figure 1 - planche I) est constituée de plateaux, de plaines alluviales avec des paysages agraires faits de bocages et d'openfield. L'élevage domine malgré une expansion des zones de culture (maïs). Le mitage urbain progresse également en bordure de Guéret et des bourgs-centres.

Une zone de transition au centre et au centreest jusqu'à la ligne Bourganeuf-Aubusson-Auzances (figure 1 - planche I) qui est marquée par des altitudes plus élevées, constituées de collines aux versants raides entaillées par les rivières venant des hauts plateaux. La présence de la forêt s'accentue, l'élevage devient très majoritaire au cœur des structures bocagères qui dominent. Les zones de culture occupent les parties plus élevées des plaines et les replats des alvéoles (Valadas, 1990).

La partie sud se compose de paysages marqués par des reliefs élevés entrecoupés de vallées assez profondes, de structures en alvéoles aux versants raides qui préfigurent le plateau de Millevaches.

Cette organisation physique se répercute fortement dans l'organisation de l'habitat et les voies de communication.

- Une organisation qui reste fortement humanisée malgré les contraintes physiques assez élevées. L'agriculture demeure le principal secteur d'activité comme en témoigne l'importance des structures bocagères (Périgord, 1996). L'industrie qui a joué un rôle essentiel dans l'économie présente aujourd'hui un double visage fait de bâtiments abandonnés (Bosmoreau-les-Mines, Lavaveix-les-Mines) et d'un artisanat qui perdure (Tapisserie d'Aubusson, ateliers de taille de pierre, fabrication de Bardeaux, pelleterie...). Ces paysages très humanisés sont aussi le reflet d'une tradition hospitalière et d'accueil, ce qui a contribué à revitaliser certains villages, notamment au cours de ces deux dernières décennies avec l'arrivée de néo-ruraux et d'étrangers (anglais).

Ce découpage met en évidence le caractère répétitif et séquentiel d'une série d'éléments constitutifs des paysages creusois (figures 1 et 2 - planches I et II) et agissant comme des marqueurs territoriaux du département. Certains présentent une forte empreinte visuelle tels que les villages, la ruralité, les pâtures (figures 3 et 4B), les haies, les chaos de pierre (figure 5B), les zones humides, les tourbières (figure 4A), les landes, les forêts qui dépendent fortement de l'organisation générale du relief constitué de plateaux, d'alvéoles (figure 4B) et de vallées.

D'autres caractères, à dimension plus sociale, agissent peu sur le paysage visuel tout en étant fortement constitutifs de l'identité du territoire. Ils se trouvent alors symbolisés par des marqueurs territoriaux exprimant la solidarité, l'accueil, l'esprit de résistance (figure 4C), l'engouement associatif et culturel (figure 5F), le patrimoine historique et religieux (figures 4D, 5A, 5C), l'innovation (figure 5E), mais aussi la déprise économique, l'exode (figures $4 \mathrm{E}$ et $4 \mathrm{~F}$ ). Ils jouent un rôle fort dans la construction de l'image du territoire et se retrouvent beaucoup dans notre sélection (annexe 2).

\section{Des paysages favorisant un cadre de vie de qualité}

Les habitants considèrent le paysage comme une composante essentielle du cadre de vie et de leur environnement.

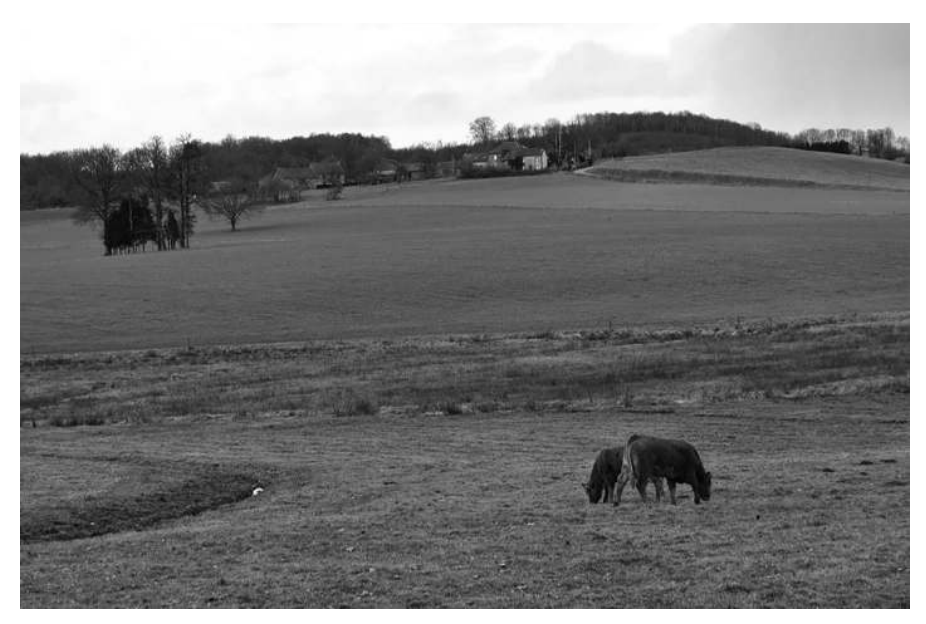

Figure 3 : Paysage agraire au cœur d'une structure alvéolaire An agrarian landscape within a granitic honeycomb structure 


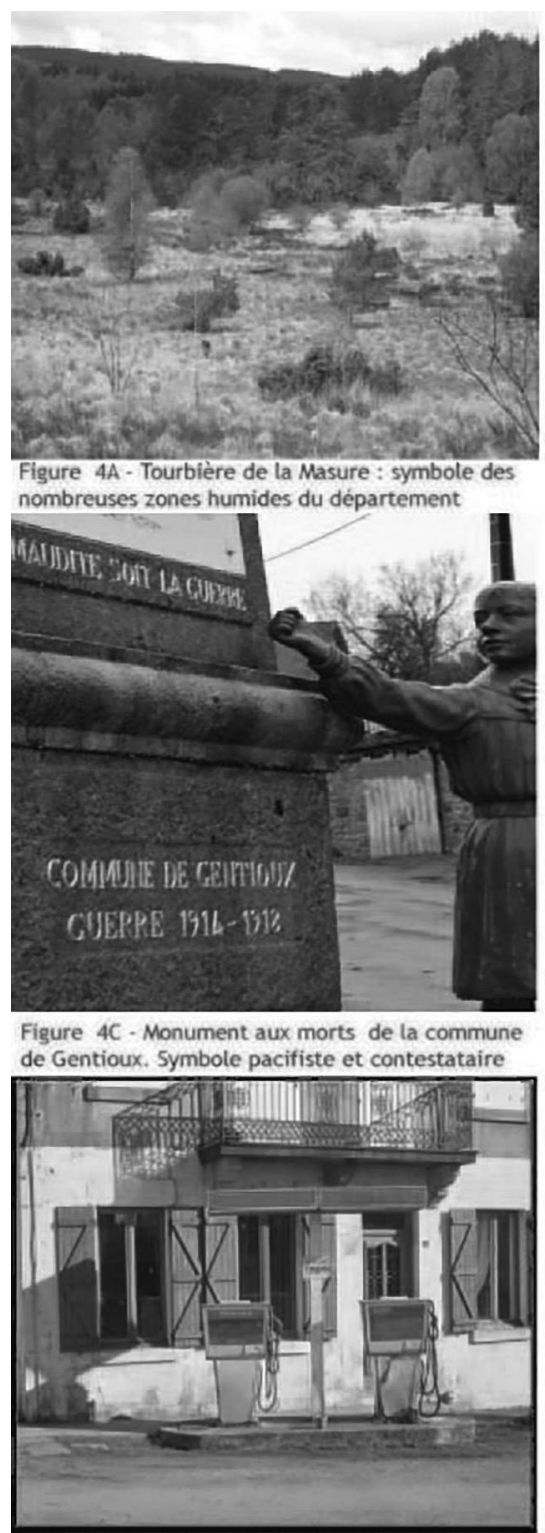

Figure $4 \mathrm{E}$ - Station abandonnée. Signe de la deprise économique et de l'exode

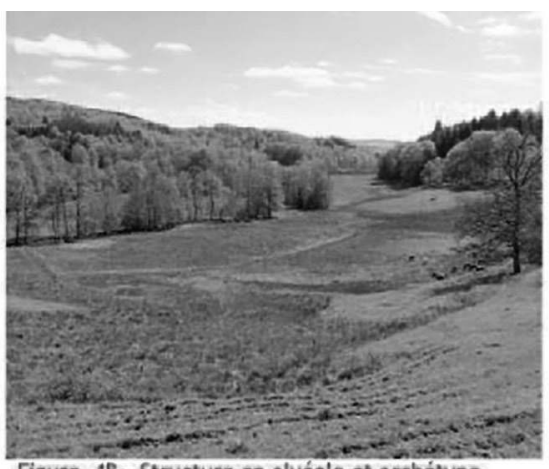

Figure $4 \mathrm{~B}$ - Structure en alvéole et archétype

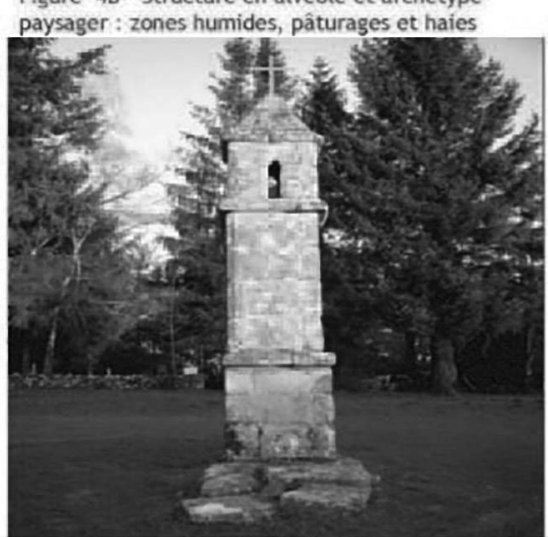

Figure 4D - Lanterne des morts. Signe religieux et patrimoine symbolique de la région Limousin

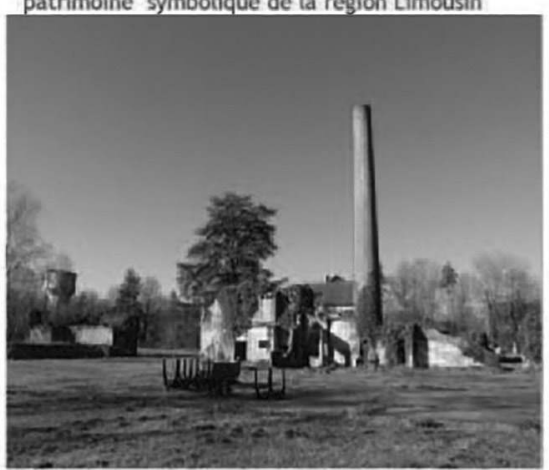

Figure 4F - Mines abandonnées à Bosmoreau - les Mines. Signe de la déprise économique et de lexode
Figure 4 : Signes paysagers et paysages symboliques landscapes

\section{La Creuse : un département «bleu, vert, calme et naturel»}

Les premiers termes employés par les enquêtés pour qualifier les paysages creusois sont dédiés aux ressources «naturelles » très présentes à leurs yeux. En référence à l’impressionnant réseau hydrographique départemental, le terme bleu est cité par 43,6\% des enquêtés alors que le vert suit avec $38,4 \%$ des citations que l'on peut associer pour partie à une vision boisée de l'espace $(43,6 \%$ des citations), bien que la forêt ne soit pas tou- jours verte! Si l'utilisation du descriptif «vert » semble adaptée à la palette paysagère produite par les pâturages, les haies bocagères et les espaces forestiers, le qualificatif bleu quant à lui pose question. Il accrédite l'omniprésence de l'eau sur un espace où chaque petit vallon porte une source, un ruisseau, une rivière et des plans d'eau, mais sa couleur est rarement bleue. Cette forte représentativité des ressources naturelles, ajoutée aux faibles densités de population, concourt à la construction d'un cadre de vie privilégié. La Creuse est perçue 
Figure 5 : Signes paysagers et paysages symboliques symbolic landscapes

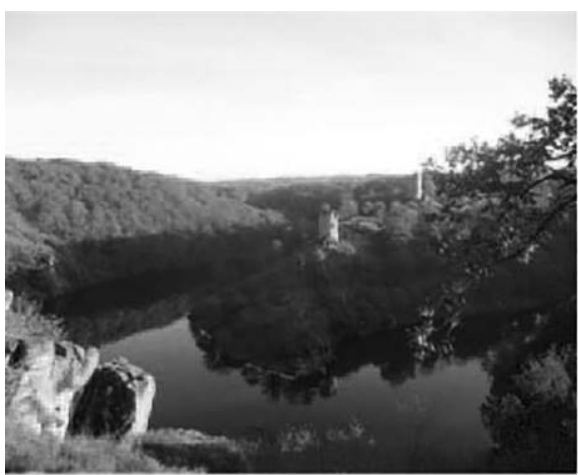

Figure $5 \mathrm{~A}$ - Ruines de Crozant : patrimoine médiéval et symbole de l'école picturale de Crozant

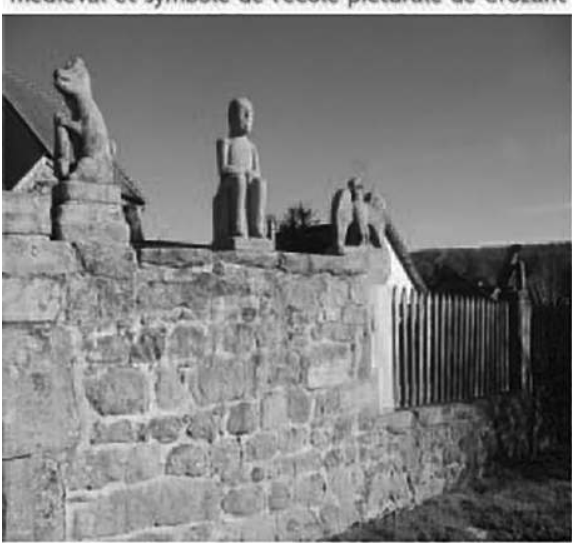

Figure 5C - Village sculpté de Masgot, symbole des savoir-faire des maçons creusois

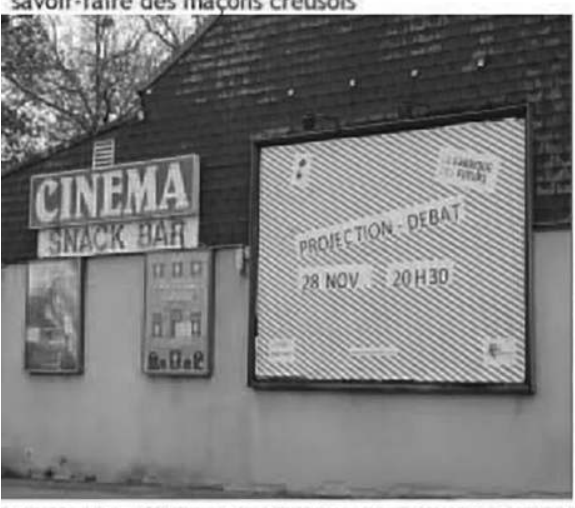

Figure 5E - Affiche "La fabrique des futurs". Symbole dinnovation dans lélaboration du projet de territoire

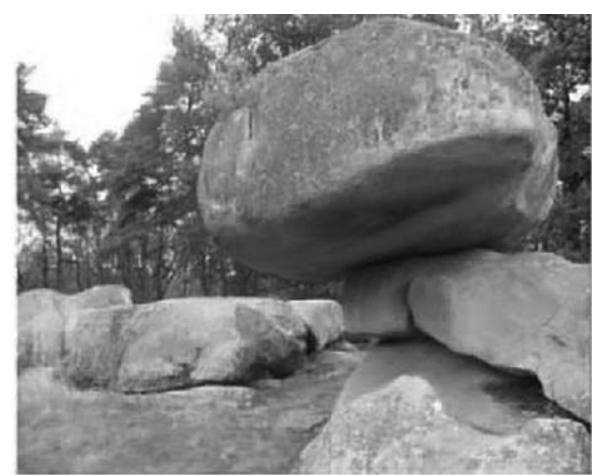

Figure 5B - Pierres Jaumâtres : symbole des chaos granitiques creusois

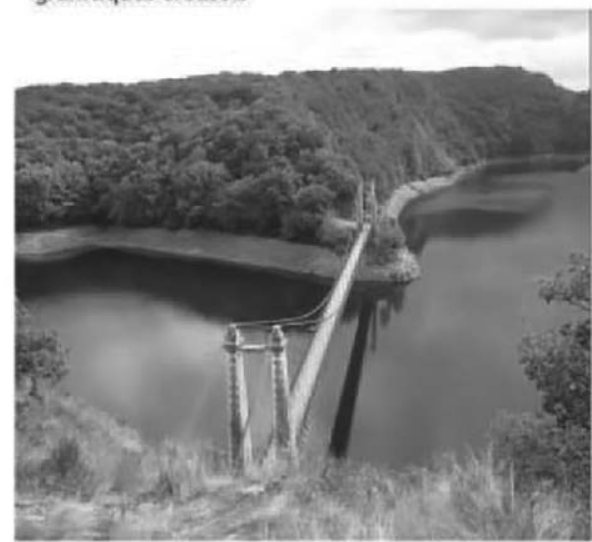

Figure 5D - Pont suspendu de Saint Marien, patrimoine architectural et signe de modernisme

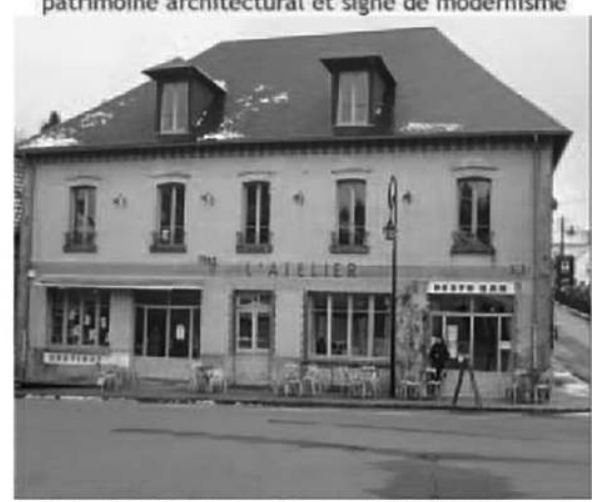

Figure 5F- L'Atelier 23. Symbole du dynamisme associatif et culturel en Creuse
« naturelle » à hauteur de 23,1\% des citations, calme $(19,7 \%)$, préservée $(19,1 \%)$, magnifique (15\%), et bénéficiant d'un cadre de vie de qualité $(6,5 \%)$. Ce sentiment de «nature » s'explique par l'omniprésence des « référents naturels » (végétation, eau, roches) même si, comme nous invite à le faire Hugonie (2003), l'usage des guillemets est de mise au mot «nature » tant celle-ci a été modifiée et se trouve sous l'emprise des hommes. La Creuse ne déroge pas à la règle, les systèmes écologiques de la planète ont tous été anthropisés, « la nature vierge est un mythe et nous vivons entourés de paysages façonnés par des générations de paysans » (Larrère et Larrère, 2009). Pour autant, les activités humaines n'ont pas toutes le même impact environnemental et visuel et - pour ce cas précis des paysages agraires, très présents en Creuse leur perception reste très positive. 


\section{La Creuse : "d'abord des paysages ruraux, agricoles et authentiques... »}

Le paysage est considéré « agricole » par 32,9\% de l'échantillon et « rural » par $19 \%$ à travers les termes de " campagne », « bourgs ", « hameaux », « villages dispersés », «petites villes », «peu peuplé » et « faible densité ». Ce qui plaît dans le caractère rural de ces paysages, c'est cette forme de permanence des structures agraires depuis le Moyen Âge. Décrits par B. Valadas en 1990, ces paysages semblent résister au temps qui passe avec une organisation adaptée des finages aux modelés des alvéoles (figure 3). Les habitats sont placés traditionnellement sur les parties supérieures, sur des terrains secs et ensoleillés sans être trop éloignés des sources alors que les parties basses des alvéoles, plus humides, sont dédiées à l'élevage; les versants selon la configuration des pentes porteront des champs de fauche ou des pâturages. Les parties les plus escarpées ou aux sols moins profonds sont boisées et de plus en plus enfrichées.

Cette ruralité est vue comme un gage de qualité de vie (apaisement, tranquillité, repos...) dans la mesure où les structures paysagères - héritées du Moyen Âge dans leur organisation des finages bocagers - relèvent du maintien des systèmes agraires (Valadas, 1990) comme la preuve de cette improbable harmonie entre les hommes et leur environnement. Ces paysages témoignent d'une pratique agricole au modernisme mesuré et où l'homme a su exploiter au mieux les caractéristiques physiques. Il en découle un paysage " authentique " très éloigné des champs à perte de vue, voués à la grande culture où les plantations de maïs, de colza, de blé alternent successivement en fonction des consignes de la Politique Agricole Commune et des cours de l'Organisation Mondiale du Commerce (PerezVitoria, 2010). Inversement, la survivance des haies bocagères au remembrement, si caractéristiques des paysages creusois, atteste du maintien du système agraire et des fonctions écologiques auxquelles elles sont associées. Cet aspect figé rassure tant pour la " conservation des repères visuels " (Donadieu et al., 2002) qu'il atteste de la possibilité de maintenir en place des systèmes socio-environnementaux en équilibres depuis des millénaires. Le cas des haies est tout à fait singulier car leur intérêt agro-écologique et les statuts de protection dont elles bénéfi- cient (code rural, code de l'urbanisme), renforcent leurs valeurs symboliques et identitaires. Longtemps associées à une ruralité du passé, elles intègrent désormais les valeurs symboliques des pratiques agro-écologiques d'aujourd'hui et de demain.

\section{En Creuse, quelques lieux emblématiques et un tout unique}

Les habitants de la Creuse ont fait l'objet de questions spécifiques cherchant à identifier une géographie paysagère de leurs lieux sensibles et intimes ainsi que des lieux qu'ils estiment être emblématiques et reconnus comme tels.

Toutes ces investigations ont confirmé l'absence de lieux remarquables et identifiés comme tels. Le lieu le plus cité par les Creusois, à hauteur de $30 \%$, est Aubusson. Une ville qui puise sa notoriété dans les métiers à tisser avec son classement au patrimoine immatériel de l’Unesco et la récente ouverture en 2016 de la Cité Internationale de la Tapisserie d'Aubusson qui est un centre moderne de création et de formation. Le lac de Vassivière, quant à lui, serait pour $13 \%$ des répondants un des lieux caractéristiques du département. Il est le premier pôle touristique et sportif de la région avec une offre diversifiée, telle que le Centre international d'art du paysage de Vassivière. Suivent les sites de Crozant $(9,5 \%)$ pour son héritage médiéval et pictural, Senoueix $(5,5 \%)$ avec son pont de pierre en forme d'arche, et les Pierres Jaumâtres $(5,2 \%)$, l'un des plus beaux chaos granitiques de France (figures 5A, 6, 5B). Il n'y a donc pas réellement de lieux emblématiques qui ressortent de cette enquête mais plutôt quelques sites singuliers qui ne sont pas partagés par tous les habitants. Par contre, nos enquêtes révèlent la description d'une forme d'archétype paysager (figure 4B) combinant à la fois les couleurs vertes des pâturages aux scintillements des rivières, et où se juxtaposent la verticalité des haies et la douceur des lignes courbes des «vallons» (33,5\% des citations). Cette absence de lieu unique se trouve donc compensée en un « tout unique » fait d'une homogénéité paysagère bâtie sur les liens étroits établis entre les hommes (activité, bâti) et les composantes «naturelles » (eau, végétation, pierres). Contrairement à toute attente, cette réalité ne porte en rien préjudice à l'image du territoire par les Creusois, au contraire. 
Figure 6 : Regards et atmosphères variés du pont de Senoueix.

A variety of views and atmospheres around Senoueix bridge

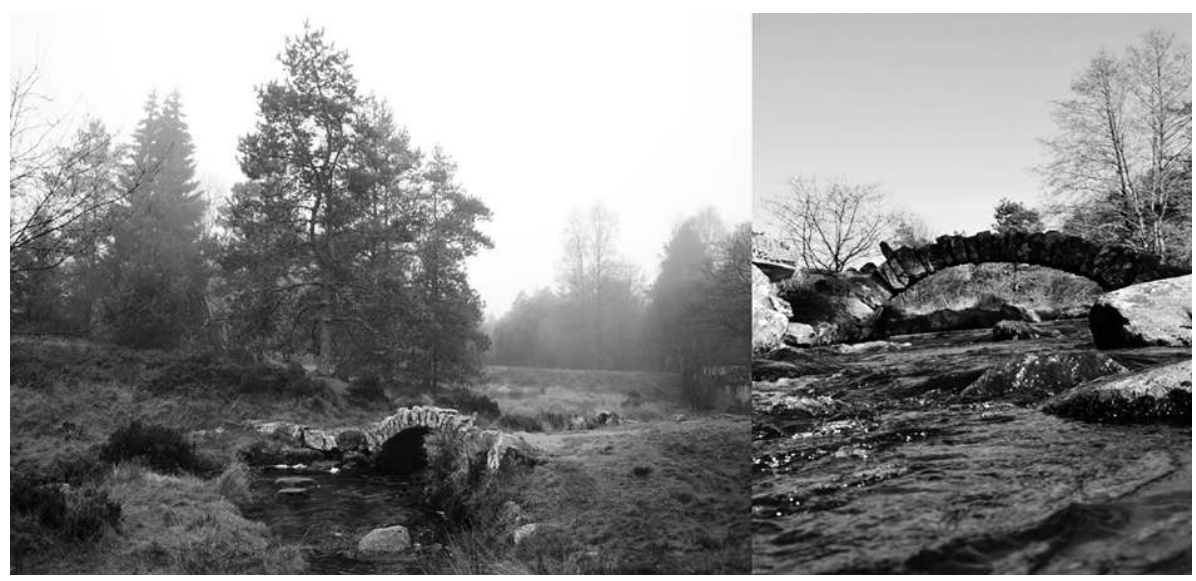

C'est une vision à la fois construite et idéelle d'un espace «à part », « en dehors » qui se trouve matériellement protégé par ses haies et en fait une sorte de « cocon », « d'îlot ». Une image idyllique qui n'est pas forcément totalement partagée à l'extérieur.

\section{La Creuse vue de l'extérieur : entre nature préservée et réalité économique}

Les personnes extérieures ont relativisé cette apparente harmonie en mettant en avant le caractère très rural du territoire.

Les principaux éléments de description donnés par les personnes extérieures à la Creuse sont «rural » $(41 \%)$, « désertique » $(38,8 \%)$ et « naturel » (32\%), des qualificatifs qui mettent en évidence une réalité socio-économique qui se traduit par son caractère rural et agricole, de faibles densités de population et par voie de conséquence un très fort ressenti «naturel ». Ce dernier sentiment englobe de nombreuses "aménités » (Donadieu et al., 2002) qui semblent bien identifiées tels que des " espaces verdoyants », des «lacs », des « haies ». Ces aspects sont associés pour $16 \%$ d'entre eux à « une qualité de vie, des densités faibles, un coût de la vie moindre, un sentiment de sécurité... ». La Creuse bénéficie ainsi d'une image très positive pour les loisirs, les vacances en famille dans un cadre naturel privilégié. Ce sentiment est notamment favorisé par une image positive de ses habitants, son caractère authentique $(6,5 \%)$ et sa capacité à prendre le temps de vivre, sans occulter pour autant son dynamisme associatif voire militant $(7 \%)$. Si le patrimoine historique demeure modeste à l'échelle nationale, il est présent en quantité sur tout le département (châteaux, maisons traditionnelles, patrimoine vernaculaire...).

Ils sont près de $25 \%$ à considérer la ruralité comme étant trop marquée et source de «vide » en termes de "population », de " services », de " manque de dynamisme ", de "diversité paysagère ». Une image qui serait notamment véhiculée par « les médias », le « bouche à oreille », des allégations qui prennent tout leur sens peu après la parution de l'article très controversé « la bouse ou la vie » publié par Technikart ${ }^{1}$. et du conflit médiatique qui s'en est suivi.

Ils sont autant à regretter un manque d'attractivité, que ce soit pour vivre ou pour travailler, en particulier chez les jeunes. Un peu plus de $12 \%$ indiquent qu'il existe trop peu d'activités ou qu'elles sont trop éloignées les unes des autres. Ce déficit vaudrait notamment pour les sites touristiques et les activités culturelles. Enfin, certains pensent que le département est méconnu, qu'il souffrirait d'un manque d'identité propre ou de figure charismatique, à même de le promouvoir et de le faire connaître.

\section{Entre regards endogènes et exogènes : des réalités vécues et idéalisées}

Globalement, les habitants de la Creuse nous ont livré un regard coloré de leur territoire (boisé, vert, bleu), à la topographie douce (vallonnée) et au cadre

\footnotetext{
1. «La bouse ou la vie : manuel de survie pour branchés de la campagne. Enterré dans le Limousin, Guéret est une ville quasi morte où il n'y a pas de fac, ni de FRAC mais des ploucs, des viocs, des bovins en surnombre et... des jeunes, qui malgré tout, n'ont pas toujours mauvais goût? Reportage au milieu de nulle part ». Technikart publié le 27 mai 2012 à $11 \mathrm{~h} 16 \mathrm{mn}$. [https://www.technikart.com/la-bouse-ou-la-vie/].
} 
naturel (bois, forêt...) et paysager préservé (20\%). Toutefois, ce regard « endogène » révèle des perceptions nuancées entre les habitants natifs ou non natifs du territoire. Par exemple, les natifs ont une vision «socio-économique » et « productive » des ressources naturelles (boisé, agricole, rude) alors que la « qualité du cadre de vie », bien qu'importante à leurs yeux, apparaît secondaire (calme). Ces regards endogènes au territoire sont ceux « des groupes sociaux qui les produisent, les utilisent, et le plus souvent les habitent ", ce qui induit leur attachement et constitue la mémoire des lieux (Donadieu et al., 2002). Les nouveaux habitants, assez fortement représentés dans notre enquête (néo-ruraux, anglais...) livrent une image plus idéalisée de la Creuse et de ses campagnes où les aménités sont largement mises en avant (vert, bleu, naturel, isolée...), ce qui s'explique aussi par un choix assumé et revendiqué « d'être venu vivre » en Creuse. Les regards de ces nouveaux habitants jouent un rôle important dans la construction des représentations endogènes car enrichies d'autres bases comparatives (ex-citadin, venus d'autres régions...) et n'hésitant pas à partager leur perception avec de nouveaux outils (multimédias). Ces derniers, souvent investis dans la vie culturelle locale, se sont associés de bon gré à notre démarche en espérant contribuer à « améliorer » l'image du département. Sentiment corroboré par les résultats de notre enquête qui révèle que 50 \% des Creusois estiment que les «non-Creusois » ont une image « négative » de leur département.

Pour autant, ce sentiment ne se vérifie pas dans notre enquête où $36 \%$ des «non-Creusois » déclarent avoir une image positive de la Creuse, contre $17 \%$ seulement d'avis négatifs. Un résultat plutôt avantageux qui s'explique par le fait qu'une majorité des avis positifs est donnée par des personnes ayant déjà visité la Creuse et ayant choisi leur destination pour ses qualités (cadre naturel, calme), ce qui nous invite encore une fois à nuancer la portée de nos résultats suite à nos échantillons qui sont peu représentatifs de la population (creusoise et française). L'autre constat met en relief une autre réalité, $43 \%$ des enquêtés ont une image neutre (ni positive ni négative) et $10 \%$ sont sans avis, ce qui voudrait dire que la campagne creusoise ne ferait plus autant rêver...
La mise en correspondance entre les résultats de la monographie et les enquêtes témoigne bien de la complexité à appréhender la construction paysagère et à en faire ressortir des idées communes. Si les similitudes sont nombreuses entre la dimension réelle de notre monographie et les perceptions vécues, c'est avant tout lié à notre approche géographique qui a privilégié des composantes physiques facilement partageables avec les habitants : modelé du relief, hydrographie, forêts, haies, bâti... Les enquêtes quant à elles traduisent des représentations issues d'une construction individuelle et collective amenant à des interprétations subjectives. En ce sens, les Creusois révèlent leur attachement à une nature protégée et protectrice (cocon, paisible, à part, tranquille, sereine, calme..) vis-à-vis d'un monde extérieur incertain, dangereux (insécurité, pollution) alors que les «non-Creusois »usant d'autres bases de comparaison - voient dans cet espace « à part » une opportunité à y pratiquer certaines formes de tourisme, mais aussi la faiblesse des autres activités économiques (vide, crise, désuet, arriéré) limitant ainsi l'attractivité globale de ce territoire.

Notre travail, à l'instar de nombreuses autres approches, témoigne une fois de plus de la subjectivité du sujet et de la quasi-impossibilité à dégager des valeurs paysagères communes permettant la sélection de paysages emblématiques et/ou identitaires. Ils sont le reflet d'une réalité issue de protocoles aux choix discutables et de perceptions individuelles dans un système influencé par la pensée collective. La question paysagère étant avant tout un sujet de sensibilité, nous souhaitons maintenant interroger l'existence de valeurs sensorielles communes.

\section{Pour une APPRoche MULTisenSORIELLE DES PAYSAGES}

L'approche exclusivement visuelle du paysage, situant le spectateur à distance de l'objet, ne suffit plus aujourd'hui à en appréhender la complexité. Les travaux de Murray-Schafer (2010) montrant la manière dont les ambiances sonores caractérisent les lieux (paysage sonore) ainsi que les travaux de Corbin (1994) sur la perception olfactive des espaces ont bouleversé la manière d'appréhender le paysage. Le paysage ne sera plus vu à distance mais vécu de l'intérieur : l'espace n'est plus 
seulement regardé mais ressenti. Cette approche " haptique de l'espace place l'individu dans une spatialité du proche, du contact et de la participation avec l'environnement extérieur qui est compris lui-même comme complexe, c'est-à-dire comme une ambiance composée de plusieurs dimensions sensorielles (sonores, tactiles, olfactives, visuelles, etc.) qui interagissent en réalité et dans laquelle le corps est comme plongé »(Besse, 2010).

Considérant que le paysage est avant tout une expérience personnelle et sensible qui dépasse le simple cadre de son interprétation visible, nous pouvons nous demander si de telles expériences peuvent faire émerger des ambiances sensorielles emblématiques d'un lieu? Afin de décrire ces ambiances, chaque étudiant et enseignant s'est vu octroyer la possibilité de rédiger un ou plusieurs textes sur des lieux de leurs choix. La démarche, relativement libre, supposait de nombreuses immersions dans les sites sélectionnés et présélectionnés afin d'en appréhender les ambiances lumineuses, sonores..., nécessaires à l'inspiration rédactionnelle.

Le paysage devient une aventure sensible, une expérience individuelle et corporelle mobilisant l'ensemble des sens et des sensations. Cependant, cette expérience individuelle s'est vite trouvée confrontée à d'autres marqueurs visibles et non visibles d'un espace qui - en participant à la construction d'un lieu - transcendent nos affects et agissent sur notre perception.

\section{Des « marqueurs territoriaux » de l'identité d'un territoire}

Notre sélection paysagère a permis de mettre en évidence «des lieux identitaires » (annexe 2), c'està-dire des lieux où l'histoire des hommes a laissé des traces visibles et non visibles qui, sous forme matérielle ou mémorielle, constituent des « marqueurs » forts, contribuant à façonner son image et son identité. Toutes ces traces constituent un ensemble de « témoignages » faits d'empreintes (visibles) auxquels s'ajoutent les récits oraux et narratifs faisant de lui un « espace dramatisé, légendé, un espace qui suscite intérêt, curiosité, source d'énigme » (Urbain et al., 2010).

Parmi les traits constituant l'identité creusoise, certains (sociaux) faiblement observables peuvent agir dans la perception des lieux et influencer son appréhension visuelle et sensorielle. Le regard « informé », en devenant sélectif dans la recherche d'éléments identitaires et « reconnus », renforce le caractère émanent et construit des lieux. Si nous prenons l'esprit de résistance et de contestation, si souvent évoqué pour caractériser la Creuse, nous constatons qu'il repose sur quelques lieux et faits devenus incontournables. Le monument aux morts de Gentioux, peut-être le plus connu, présente un enfant, orphelin de guerre, le poing levé en direction de la liste des soldats morts durant la première guerre mondiale, avec pour inscription « maudite soit la guerre » (figure 4C). Pas de gloire aux sacrifiés de la patrie ici mais un monument accusateur et pacifique qui ne sera d'ailleurs jamais officiellement inauguré. Ce monument constitue un symbole fort pour la Creuse et pour les associations pacifistes qui viennent chaque année s'y recueillir le 11 novembre. Non loin de là, à Royère-de-Vassivière, se trouve la tombe de Félix Baudy, soldat fusillé pour l'exemple en 1915 et portant une plaque où il est inscrit «Maudite soit la guerre - maudits soient les bourreaux - Baudy n'est pas un lâche - mais un martyr », reflétant la double injustice dont a été victime le soldat.

Pour ces deux exemples, l'incidence paysagère en tant que «paysage vu » est quasi-nulle, par contre l'action sociale qui a conduit ces revendications et les actuelles commémorations donne une dimension symbolique qui dépasse largement le lieu de l'action. Le regard informé sur ces faits, même en l'absence de référence visuelle, conduit à une "mise en situation » de la perception des lieux et des paysages.

Lorsque le photographe Thierry Girard évoque sa série «Histoires possibles, paysages probables ", qu'il réalise parallèlement à « Paysages insoumis ", il nous livre qu'il a "évité toutes les stèles, vestiges et autres rappels précis de l'Histoire », en ce sens « il s'en remet aux endroits où la providence nous conduit, auxquels aucun événement connu ou répertorié ne peut être attaché, mais où semble souffler malgré tout quelque esprit des lieux et où l'épaisseur, encore plus énigmatique, semble l'emporter sur toute autre considération. » Le photographe cherche, un peu à la manière des peintres, à transcender le cadre visible pour tenter de saisir l'intimité profonde et la part la plus mystérieuse du motif. Il évoque aussi les résistances du paysage à se livrer et à confier son « imago »... l'obligeant à revenir, 
parfois plusieurs fois, pour résoudre cette énigme. Dans ce projet, il confie que "la précision documentaire » constitue, pour lui, une sorte « d'alibi » pour «aller voir » ce qui est « souvent bousculé par la fiction ». L'ensemble de la démarche semble tout à fait illustrer la complexité avec laquelle il est possible d'appréhender un lieu et son paysage visible. Celui-ci est associé à une histoire qui a parfois laissé des traces, visibles ou non, qui peuvent être écrites, ou mémorielles, mais c'est aussi un lieu producteur de sensations, de ressentis. Le paysage fait vibrer quand il est porteur de sens pour celui qui, à défaut de savoir, imagine, ressent, interprète à sa manière comme une réalité qu'il fait sienne. L'interprétation relève de l'individu et de son imaginaire, même si celle-ci peut difficilement se soustraire au contexte socio-historique.

\section{Sonorité et écriture : pour une approche sensorielle des paysages emblématiques}

Afin de favoriser cette appréhension personnelle et sensible des lieux, de nombreuses immersions sur le terrain ont été réalisées. Le sens auditif a fait l'objet d'un traitement particulier tant pour sa capacité à établir une relation directe entre notre oreille « interne » et un environnement sonore « extérieur », que pour la possibilité d'effectuer des enregistrements sonores. Ces derniers ont été ensuite sélectionnés afin d'être intégrés à notre projet de livre (sous forme de CD), l'objectif étant de faire partager aux lecteurs ces ambiances sonores. Les autres sens, quant à eux, ont été exprimés par les textes lorsque les auteurs l'ont jugé utile.

La question de l'environnement sonore a été abondamment traitée depuis les années 1970 notamment avec la parution d'un ouvrage fondateur The Tuning of the world de Murray Schafer, traduit en français par l'expression bien trouvée et vite adoptée de «paysage sonore » (soundscape). Son approche environnementaliste associe la gestion des paysages sonores à une vaste composition musicale (design sonore) où l'harmonie résonnerait au rythme de l'écologie sonore. Bien que ce concept ait été quelquefois critiqué, certains éléments ont nourri notre réflexion.

Le premier concerne sa perception de «l'environnement acoustique » d'une communauté comme une "empreinte sonore " caractéristique et bien identifiable (Murray Schafer, 2010). Nos travaux creusois démontreront bien l'existence d'un univers sonore largement «hi-fi» où il est facile de relier certains «signaux » à leurs origines (rivière, cloches des églises, tracteur, tronçonneuses...) compte tenu d'une faible superposition des sons «low- $f i$ ». Toutefois, il nous a semblé difficile de discerner $e x$ situ des sons «emblématiques » pouvant être identifiés par toute une communauté et même quelques individus, leur reconnaissance ne pouvant se passer de leur contextualisation.

Ensuite, et comme ce fut déjà évoqué par Geisler, la méthode employée par Murray Schafer pâtit de son approche compartimentée et distanciée. Son mode d'analyse des paysages sonores, à partir de plusieurs caractères (le son peut être individuel, multiple, les volumes variés) et catégories (les tonalités, les signaux et les empreintes sonores) n'est pas sans rappeler la séparation de l'objet et du sujet de l'interprétation visuelle. Les concepts de «bruit de fond» (le décor) et de «signal » (la figure) évoquent la tradition picturale avec les idées de perspectives, de scènes composées (Geisler, 2013).

Afin d'éviter une lecture sonore trop " construite ", le choix des séquences acoustiques s'est opéré selon leur capacité à accompagner notre cheminement sensoriel, la rédaction devenant cette fois nécessaire à l'expression des autres sens. Murray Schafer «situe le paysage sonore aux frontières de la recherche scientifique, entre l'observation des sociétés humaines et l'art, entre l'acoustique (étude physique des sons) et la psycho acoustique » (2010), ce qui établit la dernière passerelle de notre cheminement : l'interprétation sensible et son ouverture à l'imaginaire. Bien que cette dimension sensible du paysage puisse être, selon Sansot, une source «d'émotion " lorsqu'elle nous bouleverse (choc), ou plus simplement un «vecteur sentimental » (Sansot, 1983), elle est avant tout le résultat d'une immersion totale de notre corps dans son environnement. Fluides tactiles, particules odorantes, variations sonores transcendent le visible pour éveiller notre imaginaire et nos réminiscences. Il devient un paysage qui nous parle, qui fait sens, comme le souligne Sansot (1983), considérant que « tout spectacle ne mérite pas d'être considéré comme paysage » et «qu'il faut une rencontre du sentant et du sensible ». Partant de cette considération, chaque auteur a été libre de choisir un ou plusieurs sites 
«faisant paysages » et de livrer un ressenti intime, imaginaire, sensoriel... L'une des grandes difficultés rencontrées par les auteurs a été d'identifier ce paysage « qui fait sens » par rapport aux multiples variations paysagères dont il peut faire l'objet.

Ce champ des possibles semble infini car en perpétuel mouvement et soumis à de multiples rythmes (saisonnier, journalier) combinant une multitude d'ambiances (météorologiques, lumineuses, sonores), ce qui nous fait dire à la fin de cette expérience qu'il existe autant de paysages qu'il existe de variations paysagères et sensorielles. L'appréciation sensible des paysages relève quant à elle de l'être intime, ce qui limite considérablement ses propensions à en dégager des valeurs communes et emblématiques d'un espace.

\section{Conclusion}

Notre démarche nous a fait progresser de la connaissance du territoire (monographie) pour en sélectionner des "empreintes emblématiques ", à l'étude de ses perceptions externes et internes avant de s'achever sur une approche sensorielle.

Si notre approche monographique semblait, par son caractère "scientifique ", plus rassurante par sa capacité à exprimer un contexte géographique et une histoire sociale, il est apparu ensuite que des marqueurs territoriaux et des représentations façonnent l'image des lieux au point de provoquer une forme de sélection des attentes paysagères. La recherche des paysages emblématiques et/ou identitaire se confronte alors aux besoins de coller à une réalité sociale qui ne correspond pas forcément à la réalité visuelle. En ce sens, notre sélection a privilégié les sites dont l'empreinte historique et sociale (monuments, patrimoines) est forte au détriment de la représentativité visuelle de certaines composantes paysagères (relief, végétation...) et de l'idéal paysager qu'elles nourrissent. Ce paysage rural - tel qu'il est décrit dans notre approche monographique avec ses traits symboliques (haies, bocages, tourbières), et bien qu'il ne représente pas de lieux précis - sert d'emblème à toute une somme de lieux, ce qui constitue ce «tout unique». Ce paysage prend même une dimension archétypale par l'intermédiaire de ses habitants qui voient dans la quasi permanence de ses paysages une forme d'harmonie entre l'homme et son environnement, une sorte d'îlot bucolique préservé des contraintes liées à l'économie moderne. Les regards externes, quant à eux, sont sensibles aux aménités environnementales du territoire mais portent un regard plus réaliste et critique sur sa situation économique et démographique.

Notre approche sensible trouve également un écho favorable à une affirmation de Sansot (1983), « le paysage est tout entier en lui-même » et il ne peut «se réduire en une somme de vertus ». Son caractère construit, imagé et polysensoriel nous oblige à faire preuve d'une grande humilité dans la quête de son interprétation et, quel que soit le sérieux des protocoles établis, toute tentative de généralisation est confrontée à la grande variabilité du paysage et des sensations produites. Il semble que le caractère sensoriel et éminemment personnel inhérent à leur interprétation transcende toute tentative visant à légitimer un paysage empreint de valeurs communes et servant d'emblèmes à un territoire.

Le dernier point de notre démonstration, même si elle reste partielle, révèle la préoccupation grandissante des questions touchant à la qualité du cadre de vie et de ses enjeux environnementaux dans les considérations paysagères. La récente loi de 2016 pour « la protection de la biodiversité, de la nature et des paysages » confirme leurs interrelations et les intérêts de leur gestion commune notamment suite aux approches fonctionnelles des paysages (fonctions écologiques). En maintenant ses agrosystèmes et en offrant un cadre de vie agréable, la Creuse, terre de solidarité sociale et de contestation, apparaît également comme une terre de solidarité écologique liant l'homme à la nature par un sentiment de dépendance réciproque. Un constat propice au développement de certaines activités (loisirs, tourisme...) à une époque où la société tente de renouer le dialogue avec son environnement par des démarches cognitives et sensorielles qui ne cessent de progresser. C'est ainsi qu'à l'issue de cette étude, le Conseil Départemental de la Creuse a choisi de valoriser ces expériences sensorielles et affectives en éditant en 2016 un ouvrage à vocation patrimoniale et touristique intitulé Paysages réels, nature rêvée. 


\section{Annexe 1 - Grille paysagère}

\begin{tabular}{|c|c|c|c|c|}
\hline \multicolumn{5}{|l|}{ Grille paysagère } \\
\hline \multicolumn{5}{|l|}{ Support : lieu } \\
\hline Lieu & $\mathrm{X}$ & Y & Orientation géo. & \\
\hline Limites du champ visuel & Fermées/ouvertes & Proches/lointaines & Floues/nettes & Uniques/multiples \\
\hline Descriptif géographique & \multicolumn{4}{|c|}{ Description des éléments naturels } \\
\hline Descriptif anthropique & \multicolumn{4}{|c|}{ Description des éléments humains } \\
\hline Nombre d'entités paysagères & \multicolumn{4}{|c|}{$\begin{array}{c}\text { Il s'agit de découper le paysage que vous voyez en grandes « parties », ou ensembles, selon } \\
\text { vos critères }\end{array}$} \\
\hline Formes physiques dégagées & \multicolumn{4}{|c|}{$\begin{array}{l}\text { Un pic, une dépression avec un lac, une colline, un grand lac, une lande, une tâche d'activi- } \\
\text { tés industrielles... }\end{array}$} \\
\hline $\begin{array}{l}\text { Points remarquables et éléments } \\
\text { à valoriser ou non }\end{array}$ & \multicolumn{4}{|c|}{ Paysages singuliers et remarquables, valorisés ou non. } \\
\hline Axes structurants & \multicolumn{4}{|c|}{ Routes, rivières, lignes... } \\
\hline \multicolumn{5}{|l|}{ Perception : ressenti } \\
\hline Ouïe (bruits) & \multicolumn{4}{|c|}{1 case par sens afin de n'en omettre aucun (sauf goût : facultatif) } \\
\hline \multicolumn{5}{|l|}{ Toucher (sensations physiques) } \\
\hline \multicolumn{5}{|l|}{ Odorat } \\
\hline \multicolumn{5}{|l|}{ Vue (lumière) } \\
\hline \multicolumn{5}{|l|}{ Humeur } \\
\hline Ressenti global & \multicolumn{4}{|c|}{ Exemple : angoisse, quiétude, plénitude, un cocon... } \\
\hline \multicolumn{5}{|l|}{ Météo } \\
\hline \multicolumn{5}{|l|}{ Souvenir précis } \\
\hline \multicolumn{5}{|l|}{ Interprétation : paysage } \\
\hline Mots qualificatifs & \multicolumn{4}{|c|}{ Permet de se souvenir des détails du paysage } \\
\hline Thème (lequel, pourquoi?) & & & & \\
\hline
\end{tabular}


Annexe 2 - Classement des mots clés et exemples de sites sélectionnés pour leurs paysages

\begin{tabular}{|c|c|c|c|c|c|c|c|c|}
\hline \multicolumn{9}{|c|}{ Classement par catégories } \\
\hline $\begin{array}{l}\text { Population et } \\
\text { habitat }\end{array}$ & $\begin{array}{l}\text { Société et } \\
\text { histoire }\end{array}$ & $\begin{array}{l}\text { Religion et } \\
\text { légendes }\end{array}$ & $\begin{array}{l}\text { Patrimoine } \\
\text { culturel et } \\
\text { architecture }\end{array}$ & Activités & Végétation & Relief & $\begin{array}{l}\text { Hydrogra } \\
\text { - phie }\end{array}$ & $\begin{array}{l}\text { Patrimoine } \\
\text { naturel et } \\
\text { cadre de vie }\end{array}$ \\
\hline \multicolumn{9}{|c|}{ Mots clefs issus de la monographie (éléments identitaires soulignés) } \\
\hline $\begin{array}{l}\text { village, rural, } \\
\text { exode, terre } \\
\text { d'accueil, } \\
\text { faible densité, } \\
\text { néo-ruraux, } \\
\text { anglais, } \\
\text { vacuité, } \\
\text { accessible } \\
\text { (financier), } \\
\text { vieillissement }\end{array}$ & \begin{tabular}{|l} 
crise éco- \\
nomique, \\
résistance, \\
solidaire, \\
engagement \\
politique, \\
mouvements \\
sociaux, \\
contestation, \\
maçons creu- \\
sois
\end{tabular} & $\begin{array}{l}\text { bâti et patri- } \\
\text { moine religieux } \\
\text { (églises, } \\
\text { chapelles, } \\
\text { lanternes des } \\
\text { morts, croix, } \\
\text { clochers en } \\
\text { bardeaux...) }\end{array}$ & $\begin{array}{l}\text { politique } \\
\text { culturelle, } \\
\text { innovation, } \\
\text { École de } \\
\text { Crozant, terre } \\
\text { d'artistes, } \\
\text { murs en } \\
\text { pierres sèches }\end{array}$ & \begin{tabular}{|l|} 
agriculture, \\
crise écono- \\
mique, inno- \\
vation, friches, \\
exode rural, \\
élevage, \\
fermeture, \\
rural, moderne, \\
tourisme (fami- \\
lial), \\
enclavement \\
\end{tabular} & $\begin{array}{l}\text { forêts, haies, } \\
\text { bocage, landes, } \\
\text { pâturages, } \\
\text { résineux, plan- } \\
\text { tation, } \\
\text { céréales } \\
\end{array}$ & \begin{tabular}{|l|} 
alvéoles, chaos, \\
plateaux, \\
hercynien, \\
granite, formes \\
douces, \\
Millevaches, \\
Marches
\end{tabular} & $\begin{array}{l}\text { ruisseaux, tour- } \\
\text { bières, zones } \\
\text { humides, } \\
\text { rivière Creuse, } \\
\text { étangs, dense, }\end{array}$ & $\begin{array}{l}\text { tourbières, } \\
\text { zones humides, } \\
\text { chaos, étangs, } \\
\text { rivières, } \\
\text { PNR de } \\
\text { Millevaches, } \\
\text { ZNIEFF, }\end{array}$ \\
\hline \multicolumn{9}{|c|}{ Mots clefs issus des enquêtes } \\
\hline $\begin{array}{l}\text { désert, rural, } \\
\text { refuge, vide, } \\
\text { cocon, îlot }\end{array}$ & $\begin{array}{l}\text { solidaire, } \\
\text { engagé, social, } \\
\text { hors du temps, } \\
\text { à part, incom- } \\
\text { pris, résigné }\end{array}$ & $\begin{array}{l}\text { lieux de } \\
\text { mystères et } \\
\text { légendes, } \\
\text { recueillement }\end{array}$ & \begin{tabular}{|l} 
maçons \\
creusois, \\
travail de la \\
pierre, églises, \\
Tapisseries \\
d'Aubusson,
\end{tabular} & $\begin{array}{l}\text { rural, agricole, } \\
\text { ennui, crise, } \\
\text { vide, tourisme, } \\
\text { évasion }\end{array}$ & $\begin{array}{l}\text { arbre, forêt, } \\
\text { bocage, haies, } \\
\text { vert }\end{array}$ & $\begin{array}{l}\text { pierre, granite, } \\
\text { mauvais temps, } \\
\text { brute }\end{array}$ & $\begin{array}{l}\text { rivière, eau, } \\
\text { étang, bleu }\end{array}$ & $\begin{array}{l}\text { paisible, tran- } \\
\text { quille, silence, } \\
\text { authentique, } \\
\text { ambiance } \\
\text { privilégiée, res- } \\
\text { sourcement, } \\
\text { méconnu }\end{array}$ \\
\hline \multicolumn{9}{|c|}{ Exemples de lieux et de paysages sélectionnés ( 85 au total, tous ne sont pas cités) } \\
\hline $\begin{array}{l}\text { - Village de } \\
\text { Mazeirat } \\
\text { (inhabité) } \\
\text { - Square des } \\
\text { Justes (terre } \\
\text { d'accueil) } \\
\text { - La Courtine } \\
\text { (déprise) } \\
\text { - Ligne ferro- } \\
\text { viaire abandon- } \\
\text { née Guéret/ } \\
\text { Châtre } \\
\text { - Pompes à } \\
\text { essence soli- } \\
\text { taires } \\
\text { - Devantures } \\
\text { de magasins } \\
\text { fermés } \\
\text { - Ruines de } \\
\text { Bosmoreau-les- } \\
\text { Mines } \\
\text { - Maupuy (site } \\
\text { fermé) }\end{array}$ & $\begin{array}{l}\text { - Dolmen de } \\
\text { Ponsat } \\
\text { - Village de } \\
\text { Mazeirat } \\
\text { (inhabité) } \\
\text { - Les marchés } \\
\text { creusois } \\
\text { - Le pont } \\
\text { de Senoueix } \\
\text { (xII siècle) } \\
\text { - La Courtine } \\
\text { (monument } \\
\text { dédié aux sol- } \\
\text { dats russes) } \\
\text { - École de Ma- } \\
\text { gnat-l'Etrange } \\
\text { - Bois de } \\
\text { Thouraud (lieu } \\
\text { de résistance) } \\
\text { - Monument } \\
\text { aux morts de } \\
\text { Gentioux } \\
\text { - La fabrique } \\
\text { des futurs } \\
\text { - L'Atelier 23 } \\
\text { (association } \\
\text { culturelle) }\end{array}$ & \begin{tabular}{|l} 
- Sainte \\
Radegonde \\
(chapelle) \\
- Chapelle \\
Saint Marien \\
- Chapelle \\
et vierge de \\
Péricholle la \\
Roches \\
- Cloche de \\
Saint Chris- \\
tophe \\
- Lanterne des \\
morts \\
- Chapelle \\
Blanche de \\
Felletin \\
- La Rigole \\
du Diable \\
(légende) \\
- La Pierre aux \\
neufs gradins \\
(légende) \\
- Les rochers \\
de Ménardeix \\
(Légende) \\
- Église de \\
Toulx-Sainte- \\
Croix \\
- Chapelle de \\
Jeanne d'Arc \\
(Mas Saint- \\
Jean) \\
- Le Puy des \\
trois Cornes \\
(Légende) \\
- Abbatiale \\
Saint-Pierre- \\
Saint-Paul
\end{tabular} & $\begin{array}{l}\text { - Pont } \\
\text { suspendu à } \\
\text { Budelières } \\
\text { - Viaduc de } \\
\text { Busseau } \\
\text { - Village } \\
\text { d'artistes à } \\
\text { Fresselines } \\
\text { - Lissières de } \\
\text { la tapisserie } \\
\text { (Aubusson) } \\
\text { - Les ruines de } \\
\text { Crozant } \\
\text { - La ville } \\
\text { d'Aubusson } \\
\text { - La Souter- } \\
\text { raine } \\
\text { - Notre-Dame } \\
\text { du bâtiment } \\
\text { (hommage aux } \\
\text { maçons) } \\
\text { - Village } \\
\text { sculpté de } \\
\text { Masgot } \\
\text { - Le pont de } \\
\text { Senoueix } \\
\text { (xII siècle) } \\
\text { - Barrage de } \\
\text { Chambon- } \\
\text { chard } \\
\text {-Moulin } \\
\text { à chanvre } \\
\text { (Crocq) } \\
\text { - Vassivière } \\
\text { (art contempo- } \\
\text { rain) }\end{array}$ & \begin{tabular}{|l|} 
- La ferme des \\
clautres \\
- Musée de \\
l'aviation \\
- Café \\
charcuterie à \\
Chénérailles \\
- Musée de \\
l'électrification \\
- Village \\
d'artistes à \\
Fresselines \\
- Lissières de \\
la tapisserie \\
- Ateliers \\
Oliverre \\
- Tuilerie de \\
Pouligny \\
- Pelleterie à \\
Crocq. \\
- Fermes et \\
vaches limou- \\
sines \\
- Fabrique de \\
Bardeaux à \\
Bénévent-l'Ab. \\
- Atelier Lou \\
creusois \\
- Les marchés \\
creusois \\
- Bâtiment \\
thermal \\
d'Evaux-les- \\
Bains \\
- Filature de \\
Rougnat \\
\end{tabular} & \begin{tabular}{|l} 
- Haies boca- \\
gères \\
- Forêt de \\
Chabrières \\
- La Bande \\
des Landes \\
(bocages \\
humides) \\
- Chêne Sully \\
- Domaine \\
forestier de \\
Banizette \\
\end{tabular} & \begin{tabular}{|l|} 
- Alvéoles du \\
plateau \\
- La Rigole du \\
Diable (chaos) \\
- La Pierre aux \\
neufs gradins \\
(chaos) \\
- Saint- \\
Georges-Nigre- \\
mont (relief) \\
- Pierres \\
Jaumâtres \\
- Le Puy des \\
trois Cornes \\
(chaos) \\
\end{tabular} & \begin{tabular}{|l} 
- La Celle \\
Dunoise \\
- Cascades de \\
Moulines \\
- La Gartempe \\
- Les étangs \\
de Flayat \\
- Sédelle à \\
Crozant \\
- Confluence \\
des deux \\
Creuse \\
- Étang des \\
Landes \\
- Source de la \\
Creuse \\
- Vassivière
\end{tabular} & \begin{tabular}{|l} 
- Forêt de \\
Chabrières \\
- La Celle \\
Dunoise \\
- La Bande \\
des Landes \\
(bocages \\
humides) \\
- Chêne Sully \\
- Cascades de \\
Moulines \\
- Les étangs \\
de Flayat \\
- La tourbière \\
de la Masure \\
- Domaine \\
forestier de \\
Banizette \\
- Sédelle à \\
Crozant \\
- Étang des \\
Landes \\
- PNR de \\
Millevaches
\end{tabular} \\
\hline 10 & 16 & 13 & 14 & 24 & 5 & 6 & 9 & 11 \\
\hline
\end{tabular}




\section{Bibliographie}

Berque A., 1984. Paysage-empreinte, paysage-matrice : éléments de problématique pour une géographie culturelle, Espace géographique, tome 13. p. 33-34.

Berthold R, Manville M., 2016. Paysages réels, nature rêvée, Editions du Conseil Général de la Creuse, coll. «Patrimoine de la Creuse », 54 p.

Besse J.-M., 2010. Le paysage, espace sensible, espace public, META. Research in Hermeneutics, Phenomenology and Practical Philosophy, vol.II, no 2, p. 259-286.

Corbin A., 1994. Les cloches de la terre. Paysage sonore et culture sensible dans les campagnes au XIX $X^{e}$ siècle, Paris, Albin Michel, $359 \mathrm{p}$.

Debarbieux B., 1995. Le lieu, le territoire et trois figures de rhétorique, L'Espace géographique, n 2, p. 97-112.

Di Méo G., 2002. Lidentité : une médiation essentielle du rapport espace-société, Géocarrefour, vol. 77, n² 2, p. 175-184.

Donadieu P., 2002. La société paysagiste, Actes-sud/ENSP, 96 p.

Geisler E., 2013. Du soundscape au paysage sonore, Métropolitiques, [http:/www.métropolitiques.eu/Du-soundscape-aupaysage-sonore.html].

GIRARD T., 2010. De l'esprit des lieux, URL [http://www.thierrygirard.com/textes/thg-limousin.htm].

Hugonie G., 2003. Les espaces «naturels » des français: les complexes géographiques locaux, Éditions du Temps, 256 p. Јаков M., 2008. Le Paysage, Éditions Infolio HEPIA, 192 p.

Joliet F., Beaujouan V., Jaсов M., 2004. Quelle naturalité du paysage ligérien? Exemple du Maine-et-Loire, Norois, $n^{\circ} 192$, p. 85-94.
Larrere C., Larrere R., 2009. Du bon usage de la nature: pour une philosophie de l'environnement, Paris, Flammarion, $355 \mathrm{p}$.

Leveque C., Mounolou J.-C., 2001. Biodiversité, dynamique biologique et conservation, Paris, Dunod, $272 \mathrm{p}$.

LuginbüHL Y., 2012. La mise en scène du monde : construction du paysage européen, Paris, CNRS, $432 \mathrm{p}$.

Monnet J., 2000. Les dimensions symboliques de la centralité, Cahiers de géographie du Québec, vol. 44, no 123, p. 399418.

Murray Schafer R., 2010. Le paysage sonore : le monde comme musique, Wildprojetc, $328 \mathrm{p}$.

Perez-Vitoria S., 2010. La Riposte des paysans, Paris, Actes Sud, $190 \mathrm{p}$.

Périgord M., 1996. Paysages limousins et identité régionale, Norois, $\mathrm{n}^{\circ}$ 172, p. 739-746.

Roger A., 1997. Court traité du paysage, Paris, Gallimard, $198 \mathrm{p.}$

SAnsot P., 1982. Variations paysagères, Université des sciences sociales de Grenoble, 236 p.

Urbain J.-D., 2010. Lieux, liens, légendes, Communications, no 87 , p. 99-107.

Valadas B., Franc A., 1990. Stations forestières et paysages: les granites du Massif Central, Revue forestière française, vol. 4, p. 400-416.

Veyret Y., Lemaitre A., 1996. Réflexion sur le paysage : paysage et patrimoine historique, L'information géographique, vol 60, $n^{\circ}$ 9, p. 177-183. 